\title{
MAXIMAL REGULARITY AND VISCOUS INCOMPRESSIBLE FLOWS WITH FREE INTERFACE
}

\author{
SENJO SHIMIZU \\ Faculty of Science, Shizuoka University \\ Shizuoka 422-8529, Japan \\ E-mail:ssshimi@ipc.shizuoka.ac.jp
}

\begin{abstract}
We consider a free interface problem for the Navier-Stokes equations. We obtain local in time unique existence of solutions to this problem for any initial data and external forces, and global in time unique existence of solutions for sufficiently small initial data. Thanks to global in time $L_{p}-L_{q}$ maximal regularity of the linearized problem, we can prove a global in time existence and uniqueness theorem by the contraction mapping principle.
\end{abstract}

1. Introduction. We consider a time dependent problem for the Navier-Stokes equations with free interface in a bounded domain $\Omega \subset \mathbb{R}^{n}(n \geq 2)$. For example, if a bottle with stopper half filled with water is put under zero gravity, then the air forms a bubble completely surrounded by the water. Let us consider such a model.

Let $\Omega_{t}^{+} \subset \Omega$ be occupied by the fluid of viscosity $\mu^{+}>0$ which is given only at the initial time $t=0$, while for $t>0$ it is to be determined. $\Gamma_{t}$ denotes the boundary of $\Omega_{t}^{+}$ and $\partial \Omega$ denotes the boundary of $\Omega$. $\Omega_{0}^{+}$is strictly contained in $\Omega$, namely $\overline{\Omega_{0}^{+}} \subset \Omega$ and the distance between $\Gamma_{0}$ and $\partial \Omega$ is positive. Put $\Omega_{t}^{-}=\Omega \backslash\left(\Omega_{t}^{+} \cup \Gamma_{t}\right)$. $\Omega_{t}^{-}$is occupied by the fluid of viscosity $\mu^{-}>0$. $\nu_{t}$ is the unit outward normal to $\Gamma_{t}$ of $\Omega_{t}^{+}$and $\nu_{\Omega}$ is the unit outward normal to $\partial \Omega$. We write $\Gamma=\Gamma_{0}$ and $\Omega^{ \pm}=\Omega_{0}^{ \pm}$. We assume that $\Gamma$ is a $C^{2,1}$ hypersurface while $\partial \Omega$ is a $C^{1,1}$ one.

The velocity vector field $v^{ \pm}(x, t)=\left(v_{1}^{ \pm}, \ldots, v_{n}^{ \pm}\right)^{*}$, where $M^{*}$ denotes the transpose of $M$, and the pressure $\theta^{ \pm}(x, t)$ for $x \in \Omega_{t}^{ \pm}$satisfy the free boundary problem for the Navier-Stokes equations:

2000 Mathematics Subject Classification: Primary 35Q30; Secondary 76D07.

Key words and phrases: $L_{p}-L_{q}$ maximal regularity, Navier-Stokes equations, viscous incompressible flows, free interface problem, global in time unique existence, local in time unique existence.

Partly supported by Grants-in-Aid for Scientific Research (C) - 20540164, Japan Society for the Promotion of Science. 


$$
\begin{array}{lr}
\partial_{t} v^{ \pm}+\left(v^{ \pm} \cdot \nabla\right) v^{ \pm}-\operatorname{Div} S^{ \pm}\left(v^{ \pm}, \theta^{ \pm}\right)=f^{ \pm} & \text {in } \Omega_{t}^{ \pm}, t>0, \\
\operatorname{div} v^{ \pm}=0 & \text { in } \Omega_{t}^{ \pm}, t>0, \\
{\left.\left[S^{+}\left(v^{+}, \theta^{+}\right)-S^{-}\left(v^{-}, \theta^{-}\right)\right] \nu_{t}\right|_{\Gamma_{t}}=0,} & \left.v^{+}\right|_{\Gamma_{t}}=\left.v^{-}\right|_{\Gamma_{t}} t>0, \\
\left.v^{-}\right|_{\partial \Omega}=0,\left.\quad v^{ \pm}\right|_{t=0}=v_{0}^{ \pm}, &
\end{array}
$$

where $S^{ \pm}\left(v^{ \pm}, \theta^{ \pm}\right)$is the stress tensor defined by

$$
S^{ \pm}\left(v^{ \pm}, \theta^{ \pm}\right)=\mu^{ \pm} D\left(v^{ \pm}\right)-\theta^{ \pm} I, \quad\left(D\left(v^{ \pm}\right)\right)_{j k}=\partial v_{j}^{ \pm} / \partial x_{k}+\partial v_{k}^{ \pm} / \partial x_{j},
$$

$I$ is the $n \times n$ identity matrix, and $f^{ \pm}=f^{ \pm}(x, t)$ is a given external force vector defined on $\Omega^{ \pm}$. In the model the effect of surface tension on $\Gamma_{t}$ is excluded. We assume the kinematic boundary condition:

$$
\Gamma_{t}=\{x=x(\xi, t) \mid \xi \in \Gamma\},
$$

where $x(\xi, t)$ is the solution of the Cauchy problem

$$
\frac{d x}{d t}=v(x, t),\left.\quad x\right|_{t=0}=\xi \quad \text { for } \xi \in \Gamma .
$$

This expresses the fact that the free interface $\Gamma_{t}$ consists for all $t>0$ of the same particles, which do not leave it and are not incident on it from inside $\Omega_{t}^{+}$or outside $\Omega_{t}^{-}$. Here and hereafter, given a function $w^{ \pm}$defined on $\Omega_{t}^{ \pm}$or $\Omega^{ \pm}$, we put

$$
w= \begin{cases}w^{+}(x) & x \in \Omega_{t}^{+}\left(\text {or } \Omega^{+}\right), \quad t>0 \\ w^{-}(x) & x \in \Omega_{t}^{-}\left(\text {or } \Omega^{-}\right), \quad t>0\end{cases}
$$

Moreover for a given function $w$ defined on $\Omega, w^{ \pm}$denotes the restriction of $w$ to $\Omega_{t}^{ \pm}$ or $\Omega^{ \pm}$.

If a velocity vector field $u^{ \pm}(\xi, t)$ is known as a function of the Lagrange coordinates $\xi$, then this connection can be written in the form:

$$
x=\xi+\int_{0}^{\infty} u^{ \pm}(\xi, \tau) d \tau \equiv X_{u^{ \pm}}(\xi, t) \quad \text { for } x \in \Omega_{t}^{ \pm}, \xi \in \Omega^{ \pm} .
$$

Passing to the Lagrange coordinates in (1) and setting $\theta^{ \pm}\left(X_{u^{ \pm}}(\xi, t), t\right)=\pi^{ \pm}(\xi, t)$, we obtain the initial boundary value problem on the fixed interface $\Gamma$ :

$$
\begin{aligned}
& u_{t}^{ \pm}-\operatorname{Div}\left[S^{ \pm}\left(u^{ \pm}, \pi^{ \pm}\right)+U^{ \pm}\left(u^{ \pm}, \pi^{ \pm}\right)\right]=f^{ \pm}\left(X_{u^{ \pm}}(\xi, t), t\right) \\
& \text { in } \Omega^{ \pm}, t>0 \text {, } \\
& \operatorname{div} u^{ \pm}+E^{ \pm}\left(u^{ \pm}\right)=\operatorname{div}\left[u^{ \pm}+\tilde{E}^{ \pm}\left(u^{ \pm}\right)\right]=0 \\
& \text { in } \Omega^{ \pm}, t>0 \text {, } \\
& {\left.\left[S^{+}\left(u^{+}, \pi^{+}\right)+U^{+}\left(u^{+}, \pi^{+}\right)\right] \nu\right|_{\Gamma}=\left.\left[S^{-}\left(u^{-}, \pi^{-}\right)+U^{-}\left(u^{-}, \pi^{-}\right)\right] \nu\right|_{\Gamma}} \\
& t>0 \text {, } \\
& \left.u^{+}\right|_{\Gamma}=\left.u^{-}\right|_{\Gamma},\left.\quad u^{-}\right|_{\partial \Omega}=0 \\
& t>0 \text {, } \\
& \left.u^{ \pm}\right|_{t=0}=u_{0}^{ \pm}(\xi) \\
& \text { in } \Omega^{ \pm} \text {, }
\end{aligned}
$$

where $u_{0}^{ \pm}(\xi)=v_{0}^{ \pm}(x), \nu$ is the unit outward normal to $\Gamma$ of $\Omega^{+}$, and $U^{ \pm}\left(u^{ \pm}, \pi^{ \pm}\right), E^{ \pm}\left(u^{ \pm}\right)$ and $\tilde{E}^{ \pm}\left(u^{ \pm}\right)$are nonlinear terms of the following forms:

$$
\begin{aligned}
& U^{ \pm}\left(u^{ \pm}, \pi^{ \pm}\right)=V_{1}^{ \pm}\left(\int_{0}^{t} \nabla u^{ \pm} d \tau\right) \nabla u^{ \pm}+V_{2}^{ \pm}\left(\int_{0}^{t} \nabla u^{ \pm} d \tau\right) \pi^{ \pm}, \\
& E^{ \pm}\left(u^{ \pm}\right)=V_{3}^{ \pm}\left(\int_{0}^{t} \nabla u^{ \pm} d \tau\right) \nabla u^{ \pm}, \quad \tilde{E}^{ \pm}\left(u^{ \pm}\right)=V_{4}^{ \pm}\left(\int_{0}^{t} \nabla u^{ \pm} d \tau\right) u^{ \pm}
\end{aligned}
$$

with some polynomials $V_{j}^{ \pm}(\cdot)$ such as $V_{j}^{ \pm}(0)=0(j=1,2,3,4)$. 
The linearized problem of (3) is the following:

$$
\begin{array}{lr}
\partial_{t} u^{ \pm}-\operatorname{Div} S^{ \pm}\left(u^{ \pm}, \pi^{ \pm}\right)=f & \text { in } \Omega^{ \pm}, t>0, \\
\operatorname{div} u^{ \pm}=g^{ \pm}=\operatorname{div} \tilde{g} & \text { in } \Omega^{ \pm}, t>0, \\
{\left.\left[S^{+}\left(u^{+}, \pi^{+}\right)-S^{-}\left(u^{-}, \pi^{-}\right)\right] \nu\right|_{\Gamma}=h^{+}-\left.h^{-}\right|_{\Gamma},} & \left.u^{+}\right|_{\Gamma}=\left.u^{-}\right|_{\Gamma} \quad t>0, \\
\left.u^{-}\right|_{\partial \Omega}=0,\left.\quad u^{ \pm}\right|_{t=0}=u_{0} . &
\end{array}
$$

First of all, we introduce function spaces and some symbols. For any domain $D$ in $\mathbb{R}^{n}$, integer $m$ and $1 \leq q \leq \infty, L_{q}(D)$ and $W_{q}^{m}(D)$ denote the usual Lebesgue space and Sobolev space of functions defined on $D$ with norms: $\|\cdot\|_{L_{q}(D)}$ and $\|\cdot\|_{W_{q}^{m}(D)}$, respectively. And also, for any Banach space $X$, interval $I$, integer $\ell$ and $1 \leq p \leq \infty, L_{p}(I, X)$ and $W_{p}^{\ell}(I, X)$ denote the usual Lebesgue space and Sobolev space of $X$-valued functions defined on $I$ with norms: $\|\cdot\|_{L_{p}(I, X)}$ and $\|\cdot\|_{W_{p}^{\ell}(I, X)}$, respectively. Set

$$
\begin{aligned}
& W_{q, p}^{\ell, m}(D \times I)=L_{p}\left(I, W_{q}^{\ell}(D)\right) \cap W_{p}^{m}\left(I, L_{q}(D)\right), \\
& \|u\|_{W_{q, p}^{\ell, m}(D \times I)}=\|u\|_{L_{p}\left(I, W_{q}^{\ell}(D)\right)}+\|u\|_{W_{p}^{m}\left(I, L_{q}(D)\right)}, \\
& W_{q}^{0}(D)=L_{q}(D), \quad W_{p}^{0}(I, X)=L_{p}(I, X), \\
& W_{p, 0}^{\ell}((0, T), X)=\left\{u \in W_{p}^{\ell}((-\infty, T), X) \mid u=0 \text { for } t<0\right\}, \\
& W_{p, 0}^{0}((0, T), X)=L_{p, 0}((0, T), X) .
\end{aligned}
$$

Given $\alpha \in \mathbb{R}$, we set

$$
\begin{gathered}
\left\langle D_{t}\right\rangle^{\alpha} u(t)=\mathcal{F}^{-1}\left[\left(1+s^{2}\right)^{\alpha / 2} \mathcal{F} u(s)\right](t), \\
H_{p}^{\alpha}(\mathbb{R}, X)=\left\{u \in L_{p}(\mathbb{R}, X) \mid\left\langle D_{t}\right\rangle^{\alpha} u \in L_{p}(\mathbb{R}, X)\right\}, \\
\|u\|_{H_{p}^{\alpha}(\mathbb{R}, X)}=\left\|\left\langle D_{t}\right\rangle^{\alpha} u\right\|_{L_{p}(\mathbb{R}, X)}+\|u\|_{L_{p}(\mathbb{R}, X)} .
\end{gathered}
$$

Here and hereafter, $\mathcal{F}$ and $\mathcal{F}^{-1}$ denote the Fourier transform and its inverse, respectively. Set

$$
\begin{gathered}
H_{q, p}^{1,1 / 2}(D \times \mathbb{R})=H_{p}^{1 / 2}\left(\mathbb{R}, L_{q}(D)\right) \cap L_{p}\left(\mathbb{R}, W_{q}^{1}(D)\right), \\
\|u\|_{H_{q, p}^{1,1 / 2}(D \times \mathbb{R})}=\|u\|_{H_{p}^{1 / 2}\left(\mathbb{R}, L_{q}(D)\right)}+\|u\|_{L_{p}\left(\mathbb{R}, W_{q}^{1}(D)\right)}, \\
{[2 p t] H_{q, p, 0}^{1,1 / 2}(D \times(0, \infty))=\left\{u \in H_{q, p}^{1,1 / 2}(D \times \mathbb{R}) \mid u=0 \text { for } t<0\right\} .}
\end{gathered}
$$

Finally, given $0<T \leq \infty$ we set

$$
\begin{gathered}
H_{q, p, 0}^{1,1 / 2}(D \times(0, T))=\left\{u \mid \exists v \in H_{q, p, 0}^{1,1 / 2}(D \times(0, \infty)), u=v \text { on } D \times(0, T)\right\}, \\
\|u\|_{H_{q, p, 0}^{1,1 / 2}(D \times(0, T))}=\inf \left\{\|v\|_{H_{q, p}^{1,1 / 2}(D \times \mathbb{R})}^{1,1 / 2} \mid\right. \\
\left.v \in H_{q, p, 0}^{1,1 / 2}(D \times(0, \infty)) \text { with } v=u \text { on } D \times(0, T)\right\} .
\end{gathered}
$$

Given a Banach space $X$ with norm $\|\cdot\|_{X}$, we set

$$
X^{n}=\left\{v=\left(v_{1}, \ldots, v_{n}\right)^{*} \mid v_{j} \in X\right\}, \quad\|v\|_{X}=\sum_{j=1}^{n}\left\|v_{j}\right\|_{X} .
$$

$F=\left(F_{i j}\right)$ means the $n \times n$ matrix whose $i$-th row and $j$-th column component is $F_{i j}$. For the differentiation of the $n \times n$ matrix of functions $F=\left(F_{i j}\right)$, the $n$-vector of functions 
$u=\left(u_{1}, \ldots, u_{n}\right)^{*}$ and the scalar function $\theta$, we use the following symbols: $\theta_{t}=\partial_{t} \theta=$ $\partial \theta / \partial t, \partial_{j} \theta=\partial \theta / \partial x_{j}$

$$
\begin{gathered}
\nabla \theta=\left(\partial_{1} \theta, \ldots, \partial_{n} \theta\right)^{*}, \quad u_{t}=\partial_{t} u=\left(\partial_{t} u_{1}, \ldots, \partial_{t} u_{n}\right), \quad \nabla u=\left(\partial_{i} u_{j}\right), \\
\operatorname{div} u=\sum_{j=1}^{n} \partial_{j} u_{j}, \quad \operatorname{Div} F=\left(\sum_{j=1}^{n} \partial_{j} F_{1 j}, \ldots, \sum_{j=1}^{n} \partial_{j} F_{n j}\right)^{*} .
\end{gathered}
$$

We denote by $C$ a generic constant and $C_{a, b} \ldots$ denotes a constant depending on the quantities $a, b, \ldots$. The constants $C$ and $C_{a, b, \ldots}$ may change from line to line.

We recall previous results concerning free interface problems. Tanaka [18] proved global in time solvability of $(3)$ in $W_{2}^{2+\alpha}$ with $\alpha \in\left(\frac{1}{2}, 1\right)$ for $n=3$ and sufficiently small data with surface tension. Takahashi [17] proved global in time existence of weak solutions of (1) in the space such that the first derivative of the velocity is in $L_{p}$ with $p>2(n+1)$ with respect to time and space provided that $\nu^{+}$is close to $\nu^{-}$without surface tension, based on the result of the linearized problem by Giga and Takahashi [9]. Nouri and Poupand [10] proved local in time existence of a weak solution of the Navier-Stokes equation describing a multi fluid flow for arbitrary initial data without surface tension.

When $\Omega$ is the whole space, Denisova [4] proved local in time unique solvability for arbitrary initial data in $W_{2}^{2+\alpha, 1+\frac{\alpha}{2}}$ with $\alpha \in\left(\frac{1}{2}, 1\right)$ with or without surface tension for $n=3$ by using a local in time unique solvability result for the linearized problem in [2] and [5]. Denisova and Solonnikov [7] proved local in time unique solvability for arbitrary initial data in the Hölder spaces with a power-like weight for $n=3$ with surface tension by using a local in time unique solvability result for the linearized problem in [3] and [6]. Abels [1] proved global in time existence of varifold and measure-valued varifold solutions for singular free interfaces with or without surface tension.

Our purpose of this paper is to state $L_{p}-L_{q}$ maximal regularity of (4) global in time and local in time in the anisotropic Sobolev space $W_{q, p}^{2,1}$. And we state global in time unique existence of solutions of (3) for sufficiently small initial data when the external force $f=0$, and local in time unique existence of solutions of (3) for any initial data and external forces in $W_{q, p}^{2,1}$ with $2<p<\infty$ and $n<q<\infty$. In this paper we announce our results. The proofs will be given in a forthcoming paper.

We consider the problem by using the analytic semigroup approach just as Shibata and Shimizu $[13,14,15]$. One of our main tools to show $L_{p}-L_{q}$ maximal regularity of (4) is $\mathcal{R}$-boundedness and an operator valued Fourier multiplier theorem which have recently been developed by Weis [20] and Denk, Hieber and Prüss [8]. In the rest of this section, we discuss the analytic semigroup approach to the initial-boundary value problem:

$$
\begin{aligned}
& \partial_{t} u^{ \pm}-\operatorname{Div} S^{ \pm}\left(u^{ \pm}, \pi^{ \pm}\right)=0, \quad \operatorname{div} u^{ \pm}=0 \quad \text { in } \Omega^{ \pm}, \\
& {\left.\left[S^{+}\left(u^{+}, \pi^{+}\right)-S^{-}\left(u^{-}, \pi^{-}\right)\right] \nu\right|_{\Gamma}=0,\left.\quad u^{+}\right|_{\Gamma}=\left.u^{-}\right|_{\Gamma},} \\
& \left.u^{-}\right|_{\partial \Omega}=\left.0 \quad u^{ \pm}\right|_{t=0}=u_{0} .
\end{aligned}
$$

Set

$$
J_{q}(\Omega)=\left\{u \in L_{q}(\Omega)^{n} \mid \operatorname{div} u=0 \text { in } \Omega,\left.\nu_{\Omega} \cdot u\right|_{\partial \Omega}=0\right\}
$$




$$
G_{q}(\Omega)=\left\{\nabla \pi \mid \pi \in W_{q}^{1}(\Omega), \int_{\Omega} \pi d x=0\right\} .
$$

We use the Helmholtz decomposition:

$$
L_{q}(\Omega)^{n}=J_{q}(\Omega) \oplus G_{q}(\Omega)
$$

for $1<q<\infty$, where $\oplus$ is the direct sum. Let $P_{q}$ denote the continuous projection from $L_{q}(\Omega)^{n}$ into $J_{q}(\Omega)$ along $G_{q}(\Omega)$ and we consider the resolvent problem corresponding to $(5)$ :

$$
\begin{aligned}
& \lambda u^{ \pm}-\operatorname{Div} S^{ \pm}\left(u^{ \pm}, \pi^{ \pm}\right)=P_{q} u_{0}, \quad \operatorname{div} u^{ \pm}=0 \quad \text { in } \Omega^{ \pm} \\
& {\left.\left[S^{+}\left(u^{+}, \pi^{+}\right)-S^{-}\left(u^{-}, \pi^{-}\right)\right] \nu\right|_{\Gamma}=0,\left.\quad u^{+}\right|_{\Gamma}=\left.u^{-}\right|_{\Gamma},} \\
& \left.u^{-}\right|_{\partial \Omega}=0 .
\end{aligned}
$$

Since we want to get the evolution equation only for the velocity $u$, we have to eliminate the pressure $\pi$. If we apply the divergence to the first equation, multiply the third equation by $\nu$, apply the divergence to the third equation, and multiply the first equation by $\nu_{\Omega}$ in (6), then we obtain

$$
\begin{aligned}
& \Delta \pi^{ \pm}=0 \quad \text { in } \Omega^{ \pm}, \\
& \pi^{+}-\left.\pi^{-}\right|_{\Gamma}=\nu \cdot\left[\mu^{+} D\left(u^{+}\right)-\mu^{-} D\left(u^{-}\right)\right] \nu-\operatorname{div} u^{+}+\left.\operatorname{div} u^{-}\right|_{\Gamma}, \\
& \partial_{\nu} \pi^{+}-\left.\partial_{\nu} \pi^{-}\right|_{\Gamma}=-\nu \cdot\left[\mu^{+} \operatorname{Div} D\left(u^{+}\right)-\mu^{-} \operatorname{Div} D\left(u^{-}\right)\right] \\
& \quad-2 \mu^{+} \partial_{\nu} \operatorname{div} u^{+}+\left.2 \mu^{-} \partial_{\nu} \operatorname{div} u^{-}\right|_{\Gamma}, \\
& \left.\partial_{\nu_{\Omega}} \pi^{-}\right|_{\partial \Omega}=\nu_{\Omega} \cdot \mu^{-}\left[\Delta u^{-}-\left.\nabla\left(\operatorname{div} u^{-}\right)\right|_{\partial \Omega} .\right.
\end{aligned}
$$

For $u^{ \pm} \in W_{q}^{2}\left(\Omega^{ \pm}\right)$with $u \in W_{q}^{1}(\Omega)$, under the condition $\int_{\Omega} \pi d x=0$, there exists a unique solution $\pi^{ \pm} \in W_{q}^{1}\left(\Omega^{ \pm}\right)$of $(7)$. Let us define the map $K^{ \pm}: W_{q}^{2}\left(\Omega^{ \pm}\right) \rightarrow W_{q}^{1}\left(\Omega^{ \pm}\right)$ by $\pi^{ \pm}=K^{ \pm}(u)$. By using $K^{ \pm}(u),(6)$ is reduced to the reduced Stokes equation:

$$
\begin{aligned}
& \lambda u^{ \pm}-\operatorname{Div} S^{ \pm}\left(u^{ \pm}, K^{ \pm}(u)\right)=P_{q} u_{0} \quad \text { in } \Omega^{ \pm}, \\
& {\left.\left[S^{+}\left(u^{+}, K^{+}(u)\right)-S^{-}\left(u^{-}, K^{-}(u)\right)\right] \nu\right|_{\Gamma}=0,\left.\quad u^{+}\right|_{\Gamma}=\left.u^{-}\right|_{\Gamma},} \\
& \left.u^{-}\right|_{\partial \Omega}=0 .
\end{aligned}
$$

Conversely, assume that $u$ solves (8). Then, since $\operatorname{div} u^{ \pm}$satisfies

$$
\begin{aligned}
& \left(\lambda-2 \mu^{ \pm} \Delta\right) \operatorname{div} u^{ \pm}=0 \quad \text { in } \Omega^{ \pm}, \\
& \left.\operatorname{div} u^{+}\right|_{\Gamma}=\left.\operatorname{div} u^{-}\right|_{\Gamma}, \\
& \left.2 \mu_{+} \partial_{\nu} \operatorname{div} u^{+}\right|_{\Gamma}=\left.2 \mu_{-} \partial_{\nu} \operatorname{div} u^{-}\right|_{\Gamma}, \\
& \left.\partial_{\nu_{\Omega}} \operatorname{div} u^{-}\right|_{\partial \Omega}=0,
\end{aligned}
$$

we obtain $\operatorname{div} u^{ \pm}=0$ in $\Omega^{ \pm}$. Therefore (7) is equivalent to (8).

Let us define the reduced Stokes operator $A_{q}$ :

$$
\begin{aligned}
& A_{q} u=-\operatorname{Div} S^{ \pm}\left(u^{ \pm}, K^{ \pm}(u)\right), \quad u \in \mathcal{D}\left(A_{q}\right), \\
& \mathcal{D}\left(A_{q}\right)=\left\{u \in W_{q}^{1}(\Omega)^{n} \cap J_{q}(\Omega)\left|u^{ \pm} \in W_{q}^{2}\left(\Omega^{ \pm}\right)^{n}, u^{-}\right|_{\partial \Omega}=0,\right. \\
&\left.\left.u^{+}\right|_{\Gamma}=\left.u^{-}\right|_{\Gamma},\left.\quad\left[S^{+}\left(u^{+}, K^{+}(u)\right)-S^{-}\left(u^{-}, K^{-}(u)\right)\right] \nu\right|_{\Gamma}=0\right\} .
\end{aligned}
$$

From Shibata and Shimizu [12], we know the following theorem. 
Proposition 1. Let $1<p<\infty, 0<\epsilon<\pi / 2$ and

$$
\Sigma_{\epsilon}=\{\lambda \in \mathbb{C} \backslash\{0\}|| \arg \lambda \mid \leq \pi-\epsilon\} .
$$

Then, there exists $\sigma>0$ such that for every $u_{0} \in L_{q}(\Omega)^{n}, \lambda \in \mathbb{C} \backslash(-\infty,-\sigma)$, (8) admits a unique solution $u^{ \pm} \in W_{q}^{2}\left(\Omega^{ \pm}\right)^{n}$ with $u \in W_{q}^{1}(\Omega)^{n}$ which satisfies the estimate:

$$
|\lambda|\|u\|_{L_{q}(\Omega)}+|\lambda|^{\frac{1}{2}}\|\nabla u\|_{L_{q}(\Omega)}+\sum_{+-}\left\|u^{ \pm}\right\|_{W_{q}^{2}\left(\Omega^{ \pm}\right)} \leq C_{q, \epsilon, \sigma}\left\|u_{0}\right\|_{L_{q}(\Omega)}
$$

for every $\lambda \in \Sigma_{\epsilon} \cup\{\lambda \in \mathbb{C}|| \lambda \mid \leq \sigma\}$.

Combining the fact that $A_{q}$ is a densely defined closed operator and Proposition 1 , we obtain the following proposition.

Proposition 2. Let $1<q<\infty$. Then $A_{q}$ generates an analytic semigroup $\left\{e^{-A_{q} t}(t)\right\}_{t \geq 0}$ on $J_{q}(\Omega)$ with exponential stability:

$$
\left\|e^{-t A_{q}} u_{0}\right\|_{J_{q}(\Omega)} \leq C_{\gamma, q} e^{-\gamma t}\left\|u_{0}\right\|_{J_{q}(\Omega)} \quad \text { for } \gamma>0 .
$$

2. Results. In this section, we shall state our results. For the initial data, we introduce the following space:

$$
\mathcal{D}_{q, p}(\Omega)=\left[J_{q}(\Omega), \mathcal{D}\left(A_{q}\right)\right]_{1-1 / p, p},
$$

where $[\cdot, \cdot]_{\theta, p}$ denotes the real interpolation functor. Let us define the Besov spaces $B_{q, p}^{2(1-1 / p)}\left(\Omega^{ \pm}\right)$and $B_{q, p}^{1-1 / p}(\Omega)$ by the real interpolation

$$
\begin{gathered}
B_{q, p}^{2(1-1 / p)}\left(\Omega^{ \pm}\right)=\left[L_{q}\left(\Omega^{ \pm}\right), W_{q}^{2}\left(\Omega^{ \pm}\right)\right]_{1-1 / p, p}, \\
B_{q, p}^{1-1 / p}(\Omega)=\left[L_{q}(\Omega), W_{q}^{1}(\Omega)\right]_{1-1 / p, p} .
\end{gathered}
$$

Then by Triebel [19] or Steiger [16] we obtain

$$
\begin{aligned}
& \mathcal{D}_{q, p}(\Omega)= \\
& \left\{\begin{array}{r}
\left\{u \in B_{q, p}^{1-1 / p}(\Omega), u^{ \pm} \in B_{q, p}^{2(1-1 / p)}\left(\Omega^{ \pm}\right) \mid \operatorname{div} u=0 \text { in } \Omega,\right. \\
\left.\left.u^{-}\right|_{\partial \Omega}=0,\left.u^{+}\right|_{\Gamma}=\left.u^{-}\right|_{\Gamma},\left.\quad\left[S^{+}\left(u^{+}, K^{+}(u)\right)-S^{-}\left(u^{-}, K^{-}(u)\right)\right] \nu\right|_{\Gamma}=0\right\} \\
\text { when } 2(1-1 / p)>1+1 / q, \\
\left\{u \in B_{q, p}^{1-1 / p}(\Omega), u^{ \pm} \in B_{q, p}^{2(1-1 / p)}\left(\Omega^{ \pm}\right) \mid \operatorname{div} u=0 \text { in } \Omega,\right. \\
\left.\left.u^{-}\right|_{\partial \Omega}=0,\left.u^{+}\right|_{\Gamma}=\left.u^{-}\right|_{\Gamma}\right\} \\
\text { when } 1 / q<2(1-1 / p)<1+1 / q, \\
\left\{u \in B_{q, p}^{1-1 / p}(\Omega), u^{ \pm} \in B_{q, p}^{2(1-1 / p)}\left(\Omega^{ \pm}\right) \mid \operatorname{div} u=0 \text { in } \Omega\right\} \\
\text { when } 2(1-1 / p)<1 / q .
\end{array}\right.
\end{aligned}
$$

The first theorem shows global in time $L_{p}-L_{q}$ maximal regularity of (4) with exponential decay.

TheOREM 1. Let $1<p, q<\infty$. Then there exists a $\gamma_{0}>0$ such that if $u_{0}, f, \tilde{g}, g^{ \pm}$and $h^{ \pm}$for (4) satisfy the conditions

$$
\begin{gathered}
u_{0} \in \mathcal{D}_{q, p}(\Omega), e^{\gamma t} f \in L_{p}\left(\mathbb{R}_{+}, L_{q}(\Omega)\right)^{n}, e^{\gamma t} \tilde{g} \in W_{p, 0}^{1}\left(\mathbb{R}_{+}, L_{q}(\Omega)\right)^{n}, \\
e^{\gamma t} g^{ \pm} \in L_{p}\left(\mathbb{R}_{+}, W_{q}^{1}\left(\Omega^{ \pm}\right)\right), e^{\gamma t} h^{ \pm} \in H_{q, p, 0}^{1,1 / 2}\left(\Omega^{ \pm} \times \mathbb{R}_{+}\right)^{n}
\end{gathered}
$$


for some $\gamma \in\left[0, \gamma_{0}\right]$, and

$$
\left.\nu \cdot\left(\tilde{g}^{+}-\tilde{g}^{-}\right)\right|_{\Gamma}=0, \quad \int_{\Omega} g d x=0, \quad \int_{\partial \Omega} \nu_{\Omega} \cdot \tilde{g} d \sigma=0
$$

for $t>0$, then (4) admits a unique solution

$$
\left(u^{ \pm}, \pi^{ \pm}\right) \in W_{q, p}^{2,1}\left(\Omega^{ \pm} \times \mathbb{R}_{+}\right)^{n} \times L_{p}\left(\mathbb{R}_{+}, W_{q}^{1}\left(\Omega^{ \pm}\right)\right)
$$

with $u \in W_{q, p}^{1,1}\left(\Omega \times \mathbb{R}_{+}\right)$and $\int_{\Omega} \pi d x=0$. Moreover there exist $\tilde{\pi}^{ \pm} \in H_{q, p}^{1,1 / 2}\left(\Omega^{ \pm} \times \mathbb{R}_{+}\right)$ such that $\left.\tilde{\pi}^{ \pm}\right|_{\Gamma}=\left.\pi^{ \pm}\right|_{\Gamma}$ for $t>0$. The solution satisfies the estimate

$$
\begin{aligned}
\sum_{+-}\left(\left\|e^{\gamma t} u^{ \pm}\right\|_{W_{q, p}^{2,1}\left(\Omega^{ \pm} \times \mathbb{R}_{+}\right)}+\right. & \left.\left\|e^{\gamma t} \pi^{ \pm}\right\|_{L_{p}\left(\mathbb{R}_{+}, W_{q}^{1}\left(\Omega^{ \pm}\right)\right)}+\left\|e^{\gamma t} \tilde{\pi}^{ \pm}\right\|_{H_{q, p}^{1,1 / 2}\left(\Omega^{ \pm} \times \mathbb{R}_{+}\right)}\right) \\
+\left\|e^{\gamma t} u\right\|_{W_{q, p}^{1,1}\left(\Omega \times \mathbb{R}_{+}\right)} \leq C & \left\{\left\|u_{0}\right\|_{\mathcal{D}_{q, p}(\Omega)}+\left\|e^{\gamma t} f\right\|_{L_{p}\left(\mathbb{R}_{+}, L_{q}(\Omega)\right)}+\left\|e^{\gamma t} \tilde{g}\right\|_{W_{p}^{1}\left(\mathbb{R}_{+}, L_{q}(\Omega)\right)}\right. \\
& \left.+\sum_{+-}\left(\left\|e^{\gamma t} g^{ \pm}\right\|_{L_{p}\left(\mathbb{R}_{+}, W_{q}^{1}\left(\Omega^{ \pm}\right)\right)}+\left\|e^{\gamma t} h^{ \pm}\right\|_{H_{q, p}^{1,1 / 2}\left(\Omega^{ \pm} \times \mathbb{R}_{+}\right)}\right)\right\} .
\end{aligned}
$$

The second theorem shows local in time $L_{p^{-}} L_{q}$ maximal regularity of (4).

TheOREM 2. Let $1<p, q<\infty$. If $u_{0}, f, \tilde{g}, g^{ \pm}$and $h^{ \pm}$for (4) satisfy the conditions

$$
\begin{gathered}
u_{0} \in \mathcal{D}_{q, p}(\Omega), f \in L_{p}\left((0, T), L_{q}(\Omega)\right)^{n}, \tilde{g} \in W_{p, 0}^{1}\left((0, T), L_{q}(\Omega)\right)^{n}, \\
g^{ \pm} \in L_{p}\left((0, T), W_{q}^{1}\left(\Omega^{ \pm}\right)\right), h^{ \pm} \in H_{q, p, 0}^{1,1 / 2}\left(\Omega^{ \pm} \times(0, T)\right)^{n}
\end{gathered}
$$

and

$$
\left.\nu \cdot\left(\tilde{g}^{+}-\tilde{g}^{-}\right)\right|_{\Gamma}=0, \quad \int_{\Omega} g d x=0, \quad \int_{\partial \Omega} \nu_{\Omega} \cdot \tilde{g} d \sigma=0
$$

for $t>0$, then (4) admits a unique solution

$$
\left(u^{ \pm}, \pi^{ \pm}\right) \in W_{q, p}^{2,1}\left(\Omega^{ \pm} \times(0, T)\right)^{n} \times L_{p}\left((0, T), W_{q}^{1}\left(\Omega^{ \pm}\right)\right)
$$

with $u \in W_{q, p}^{1,1}(\Omega \times(0, T))$ and $\int_{\Omega} \pi d x=0$. Moreover there exists $\tilde{\pi}^{ \pm} \in H_{q, p}^{1,1 / 2}\left(\Omega^{ \pm} \times(0, T)\right)$ such that $\left.\tilde{\pi}^{ \pm}\right|_{\Gamma}=\left.\pi^{ \pm}\right|_{\Gamma}$ for $t>0$. The solution satisfies the estimate

$$
\begin{gathered}
\sum_{+-}\left(\left\|u^{ \pm}\right\|_{W_{q, p}^{2,1}\left(\Omega^{ \pm} \times(0, T)\right)}+\left\|\pi^{ \pm}\right\|_{L_{p}\left((0, T), W_{q}^{1}\left(\Omega^{ \pm}\right)\right)}+\left\|\tilde{\pi}^{ \pm}\right\|_{H_{q, p}^{1,1 / 2}\left(\Omega^{ \pm} \times(0, T)\right)}\right)+\|u\|_{W_{q, p}^{1,1}(\Omega \times(0, T))} \\
\leq C\left\{\left\|u_{0}\right\|_{\mathcal{D}_{q, p}(\Omega)}+\|f\|_{L_{p}\left((0, T), L_{q}(\Omega)\right)}+\|\tilde{g}\|_{W_{p}^{1}\left((0, T), L_{q}(\Omega)\right)}\right. \\
\left.+\sum_{+-}\left(\left\|g^{ \pm}\right\|_{L_{p}\left((0, T), W_{q}^{1}\left(\Omega^{ \pm}\right)\right)}+\left\|h^{ \pm}\right\|_{H_{q, p}^{1,1 / 2}\left(\Omega^{ \pm} \times(0, T)\right)}\right)\right\}
\end{gathered}
$$

where $C$ is independent of $T$.

The third theorem shows global in time unique solvability of (3) for $f=0$ and sufficiently small initial data which is proved by using the contraction mapping principle based on Theorem 1.

TheOREM 3. Let $2<p<\infty, n<q<\infty$ and $f=0$. Then there exist $\epsilon, \gamma>0$ such that the following assertion holds: if

$$
u_{0} \in \mathcal{D}_{q, p}(\Omega), \quad\left\|u_{0}\right\|_{\mathcal{D}_{q, p}(\Omega)} \leq \epsilon,
$$


then (3) admits a unique solution

$$
\left(u^{ \pm}, \pi^{ \pm}\right) \in W_{q, p}^{2,1}\left(\Omega^{ \pm} \times \mathbb{R}_{+}\right)^{n} \times L_{p}\left(\mathbb{R}_{+}, W_{q}^{1}\left(\Omega^{ \pm}\right)\right)
$$

with $u \in W_{q, p}^{1,1}\left(\Omega \times \mathbb{R}_{+}\right)$and $\int_{\Omega} \pi d x=0$. Moreover there exist $\tilde{\pi}^{ \pm} \in H_{q, p}^{1,1 / 2}\left(\Omega^{ \pm} \times \mathbb{R}_{+}\right)$ such that $\left.\tilde{\pi}^{ \pm}\right|_{\Gamma}=\left.\pi^{ \pm}\right|_{\Gamma}$ for $t>0$. The solution satisfies the estimate

$$
\begin{aligned}
& \sum_{+-}\left(\left\|e^{\gamma t} u^{ \pm}\right\|_{W_{q, p}^{2,1}\left(\Omega^{ \pm} \times \mathbb{R}_{+}\right)}+\left\|e^{\gamma t} \pi^{ \pm}\right\|_{L_{p}\left(\mathbb{R}_{+}, W_{q}^{1}\left(\Omega^{ \pm}\right)\right)}+\left\|e^{\gamma t} \tilde{\pi}^{ \pm}\right\|_{H_{q, p}^{1,1 / 2}\left(\Omega^{ \pm} \times \mathbb{R}_{+}\right)}\right) \\
&+\left\|e^{\gamma t} u\right\|_{W_{q, p}^{1,1}\left(\Omega \times \mathbb{R}_{+}\right)} \leq C \epsilon .
\end{aligned}
$$

The last theorem shows local in time unique solvability of (3) for any initial data and external forces, which is proved by using the contraction mapping principle based on Theorem 2.

Theorem 4. Let $2<p<\infty, n<q<\infty$. If $u_{0}$ and $f$ satisfy the conditions

$$
u_{0} \in \mathcal{D}_{q, p}(\Omega), \quad f \in L_{p}\left(\mathbb{R}_{+}, L_{q}(\Omega)\right), \quad \nabla f \in L_{\infty}\left(\Omega \times \mathbb{R}_{+}\right),
$$

then there exists

$$
T=T\left(\left\|u_{0}\right\|_{\mathcal{D}_{q, p}(\Omega)},\|f\|_{L_{p}\left(\mathbb{R}_{+}, L_{q}(\Omega)\right)},\|\nabla f\|_{L_{\infty}\left(\Omega \times \mathbb{R}_{+}\right)}\right)>0
$$

such that (3) admits a unique solution

$$
\left(u^{ \pm}, \pi^{ \pm}\right) \in W_{q, p}^{2,1}\left(\Omega^{ \pm} \times(0, T)\right)^{n} \times L_{p}\left((0, T), W_{q}^{1}\left(\Omega^{ \pm}\right)\right)
$$

with $u \in W_{q, p}^{1,1}(\Omega \times(0, T))^{n}$ and $\int_{\Omega} \pi d x=0$. Moreover there exists $\tilde{\pi}^{ \pm} \in H_{q, p}^{1,1 / 2}\left(\Omega^{ \pm} \times\right.$ $(0, T))$ such that $\left.\tilde{\pi}^{ \pm}\right|_{\Gamma}=\left.\pi^{ \pm}\right|_{\Gamma}$ for $t \in(0, T)$. The solution satisfies the estimate

$$
\begin{aligned}
\sum_{+-}\left(\left\|u^{ \pm}\right\|_{W_{q, p}^{2,1}\left(\Omega^{ \pm} \times(0, T)\right)}+\|\right. & \left.\pi^{ \pm}\left\|_{L_{p}\left((0, T), W_{q}^{1}\left(\Omega^{ \pm}\right)\right)}+\right\| \tilde{\pi}^{ \pm} \|_{H_{q, p}^{1,1 / 2}\left(\Omega^{ \pm} \times(0, T)\right)}\right) \\
& +\|u\|_{W_{q, p}^{1,1}(\Omega \times(0, T))} \leq C\left(\left\|u_{0}\right\|_{\mathcal{D}_{q, p}(\Omega)}+\|f\|_{L_{p}\left(\mathbb{R}_{+}, L_{q}(\Omega)\right)}\right) .
\end{aligned}
$$

REMARK 1. If $f$ satisfies

$$
\begin{gathered}
f \in L_{p}\left(\mathbb{R}_{+}, L_{q}(\Omega)\right), \quad \nabla f \in L_{\infty}\left(\Omega \times \mathbb{R}_{+}\right), \\
\left\|e^{\gamma t} f\right\|_{L_{p}\left(\mathbb{R}_{+}, L_{q}(\Omega)\right)}+\left\|e^{\gamma t} \nabla f\right\|_{L_{\infty}\left(\Omega \times \mathbb{R}_{+}\right)} \leq \epsilon,
\end{gathered}
$$

then the conclusion of Theorem 3 holds.

REMARK 2. Since $W_{q}^{2}\left(\Omega^{ \pm}\right) \subset C^{1}\left(\Omega^{ \pm}\right)$when $q>n$ and $J(\xi)=1 \neq 0$, there exists a $C^{1}$ diffeomorphic map from the Lagrange coordinate $\xi$ to the Euler coordinate $x$.

Since the solution $u^{ \pm} \in W_{q, p}^{2,1}\left(\Omega^{ \pm} \times \mathbb{R}_{+}\right)^{n}$ with $2<p<\infty$ and $n<q<\infty$ of (3) is exponentially stable which is stated in Theorem 3 , by (2) we obtain

$$
\begin{aligned}
|x-\xi| & \leq \int_{0}^{t}\left\|u^{ \pm}(\cdot, \tau)\right\|_{L_{\infty}\left(\Omega^{ \pm}\right)} d \tau \leq C \int_{0}^{t}\left\|u^{ \pm}(\cdot, \tau)\right\|_{W_{q}^{1}\left(\Omega^{ \pm}\right)} d \tau \\
& \leq C\left(\int_{0}^{t} e^{p \gamma \tau}\left\|u^{ \pm}(\cdot, \tau)\right\|_{W_{q}^{1}\left(\Omega^{ \pm}\right)}^{p} d \tau\right)^{1 / p}\left(\int_{0}^{t} e^{-p^{\prime} \gamma \tau} d \tau\right)^{1 / p^{\prime}} \\
& \leq C\left\|u_{0}\right\|_{\mathcal{D}_{q, p}(\Omega)} \quad \text { for } \quad x \in \Omega_{t}^{ \pm}, \xi \in \Omega^{ \pm}
\end{aligned}
$$

which implies that $\Gamma_{t}$ does not touch $\partial \Omega$ for all $t \in(0, \infty)$ under the assumption such that $\Gamma$ is strictly contained in $\Omega$ and initial data $u_{0}$ is sufficiently small. 
Roughly speaking, our proof of Theorem 1 is divided into the following three steps. First of all, we show $L_{p}-L_{q}$ maximal regularity of solutions to the model problems in the whole space, in the half-space and in the whole space with interface $x_{n}=0$ by using the operator valued Fourier multiplier theorem due to Weis [20] and Denk, Hieber and Prüss [8]. The key observation for this is to show the $\mathcal{R}$-boundedness of the family of solution operators to such model problems.

Secondly, we consider the problem (4) with $u_{0}=g^{ \pm}=\tilde{g}=h^{ \pm}=0$ in a bounded domain $\Omega$ and we use the usual localization procedure to reduce the problem to the model problems in the whole space and the half-space. To estimate the perturbation terms appearing in this procedure we use an exponential decay estimate of the analytic semigroup which is stated in Proposition 2.

Thirdly, using a solution to the Laplace equation with zero Neumann boundary condition:

$$
\Delta z=g \text { in } \Omega,\left.\quad \frac{\partial z}{\partial \nu_{\Omega}}\right|_{\partial \Omega}=0, \quad \int_{\Omega} g d x=0,
$$

we reduce the problem (4) to the case where $\tilde{g}=g^{ \pm}=0$. After this procedure, to treat the equation (4) with non-zero $h^{ \pm}$, we use a solution to the dual problem with $u_{0}=\tilde{g}=g^{ \pm}=h^{ \pm}=0$ which is discussed in the second step.

Acknowledgements. The author wishes to thank Prof. Helmut Abels for suggesting the last part of Remark 2 during the conference "Parabolic and Navier-Stokes equations", 10-17 September 2006, at Mathematical Research and Conference Center in Będlewo.

\section{References}

[1] H. Abels, On generalized solutions of two-phase flows for viscous incompressible fluids, Interface and Free Boundaries 9 (2007), 31-65.

[2] I. V. Denisova, A priori estimates for the solution of a linear time-dependent problem connected with the motion of a drop in a fluid medium, Trudy Mat. Inst. Steklov. 188 (1990), 3-21 (in Russian); English transl.: Proc. Steklov Inst. Math. 188 (1991), 1-24.

[3] I. V. Denisova, Solvability in Hölder spaces of a linear problem concerning the motion of two fluids separated by a closed surface, Algebra i Analiz 5 (1993), 122-148 (in Russian); English transl.: St. Petersburg Math. J. 5 (1994), 765-787.

[4] I. V. Denisova, Problem of the motion of two viscous incompressible fluids separated by a closed free interface, Acta Appl. Math. 37 (1994), 31-40.

[5] I. V. Denisova and V. A. Solonnikov, Solvability of a linearized problem on the motion of a drop in a fluid flow, Zap. Nauchn. Sem. Leningrad. Otdel. Mat. Inst. Steklov. (LOMI) 171 (1989), 53-65 (in Russian); English transl.: J. Soviet Math. 56 (1991), 2309-2316.

[6] I. V. Denisova and V. A. Solonnikov, Solvability in Hölder spaces of a model initialboundary value problem generated by a problem on the motion of two fluids, Zap. Nauchn. Sem. Leningrad. Otdel. Mat. Inst. Steklov. (LOMI) 181 (1991) 5-44 (in Russian); English transl.: J. Math. Sci. 70 (1994), 1717-1746.

[7] I. V. Denisova and V. A. Solonnikov, Classical solvability of the problem on the the motion of two viscous incompressible fluids, Algebra i Analiz 7 (1995), 101-142 (in Russian); English transl.: St. Petersburg Math. J. 7 (1996), 755-786. 
[8] R. Denk, M. Hieber and J. Prüss, $\mathcal{R}$-boundedness, Fourier multipliers and problems of elliptic and parabolic type, Memoirs of AMS 166 (2003), no. 788.

[9] Y. Giga and Sh. Takahashi, On global weak solutions of the nonstationary two-phase Stokes flow, SIAM J. Math. Anal. 25 (1994), 876-893.

[10] A. Nouri and F. Poupaud An existence theorem for the multifluid Navier-Stokes problem, J. Differential Equations 123 (1995), 71-88.

[11] Y. Shibata and S. Shimizu, A decay property of the Fourier transform and its application to the Stokes problem, J. Math. Fluid Mech. 3 (2001), 213-230.

[12] Y. Shibata and S. Shimizu, On a resolvent estimate of the interface problem for the Stokes system in a bounded domain, J. Differential Equations 191 (2003), 408-444.

[13] Y. Shibata and S. Shimizu, $L_{p}-L_{q}$ maximal regularity and viscous incompressible flows with free surface, Proc. Japan Acad. Ser. A Math. Sci. 81 (2005), 151-155.

[14] Y. Shibata and S. Shimizu, On the $L_{p}-L_{q}$ maximal regularity of the Neumann problem for the Stokes equations in a bounded domain, J. Reine Angew. Math. 615 (2008), 157-209.

[15] Y. Shibata and S. Shimizu, On some free boundary problem for the Navier-Stokes equations, Differential Integral Equations 20 (2007), 241-276.

[16] O. Steiger, On Navier-Stokes equations with first order boundary conditions, Ph.D. thesis, Universität Zürich, 2004.

[17] Sh. Takahashi, On global weak solutions of the nonstationary two-phase Navier-Stokes flow, Adv. Math. Sci. Appl. 5 (1995), 321-342.

[18] N. Tanaka, Global existence of two phase non-homogeneous viscous incompressible weak fluid flow, Commun. Partial Differential Equations 18 (1993), 41-81.

[19] H. Triebel, Interpolation Theory, Function Spaces, Differential Operators, 2nd edition, Johann Ambrosius Barth, Heidelberg, 1995.

[20] L. Weis, Operator-valued Fourier multiplier theorems and maximal $L_{p}$-regularity, Math. Ann. 319 (2001), 735-758. 